\title{
Counting on Matrices
}

\author{
Sajal Mukherjee ${ }^{\dagger}$ and Sanjay Mukherjee $e^{\ddagger}$ \\ $\dagger^{\dagger}$ Department of Mathematics, Indian Institute of Science, Bengaluru, India \\ Email: shyamal.sajalmukherjee@gmail.com \\ $¥$ School of Mathematical Sciences, National Institute of Science Education and Research, Bhubaneswar 752050, India \\ Homi Bhabha National Institute (HBNI), Training School Complex, Anushakti Nagar, Mumbai 400094, India \\ Email: sanjay.mukherjee@niser.ac.in
}

Received: February 23, 2021, Accepted: June 5, 2021, Published: June 18, 2021

The authors: Released under the CC BY-ND license (International 4.0)

ABSTRACT: In this paper, we have found formulas for the number of rectangular and symmetric matrices with the line sums divisible by a given integer. As an application, we have derived an explicit formula enumerating the number of traceless $n \times n,(0,1)$ symmetric matrices having line sums divisible by a given integer, which leads to an enumeration of labeled regular graphs with $n$ vertices. Also, we have found a formula for the weighted enumerator (in terms of rows and columns) of rectangular matrices, which subsequently yields some nice identities satisfying curious reciprocity phenomena.

Keywords: Enumeration; Matrices; Polynomial; Regular graphs; Symmetric functions 2020 Mathematics Subject Classification: 05A19; 05A05; 05C30; 05C38

\section{Introduction}

Enumeration of matrices (arrays) are not new in the literature. For instance, in [2] Anand, Dumir and Gupta addressed the problem of enumerating non negative square matrices with fixed line sums. For instance, they proved that if $A(n)=\frac{H(n, 2)}{(n !)^{2}}$, then

$$
\sum_{n=0}^{\infty} A(n) x^{n}=(1-x)^{-\frac{1}{2}} \mathrm{e}^{\frac{x}{2}},
$$

where $H(n, r)$ denotes the number of $n \times n$ matrices with entries from $\mathbb{N}$ and line sums (row sums and column sums) equal to $r$. For $n=3$, they also found a generating function for $H(3, r)$. In [1] Abramson and Moser addressed the problem of counting matrices with fixed row sum and column sum. Carlitz in $[3,4]$ and Gupta in [7] considered analogous problems for symmetric matrices and found several remarkable results for particular values of $n$ and $r$. In this paper, we have also addressed similar kind of problems for rectangular and symmetric matrices.

Unlike them, we have considered matrices with line sums divisible by a fixed positive integer $k$. For instance, one can think of the problem of finding the number of $m \times n$ matrices with entries from the set $\{0,1, \ldots, q\}$ such that all the row sums and column sums are even integers. It is not difficult to see that if $q$ is odd, then one can find a pretty simple recursion which yields that the required number is $(q+1)^{(m-1)(n-1)}\left(\frac{q+1}{2}\right)^{(m+n-1)}$. But, the problem arises when $q$ is even since that simple inductive argument becomes inapplicable if the number of odd terms and the number of even terms becomes different in the given set $\{0,1, \ldots, q\}$. In this paper, we have found explicit formulas for the number of $m \times n$ matrices and the number of $n \times n$ symmetric matrices having entries from $\{0,1, \ldots, q\}$ with line sums divisible by a given integer $k$. As a consequence of the results, we have also been able to find an explicit formula enumerating the number of traceless $n \times n,(0,1)$ symmetric matrices having line sums divisible by a given integer, which leads to an enumeration of labeled regular graphs with $n$ vertices. The latter is known to follow P-recursiveness from the works of Goulden, Jackson in [6] and Gessel in [5]. But our approach does not involve the use of recursion and leads to an explicit enumeration formula.

The other half of this paper is devoted to a weighted enumeration of rectangular matrices. Let us denote the given set of integers $\{0,1, \ldots, q\}$ by $S$. Let $M_{m \times n}(S)$ be the set of all $m \times n$ matrix with entries from the set $S:=\{0,1, \ldots, q\}$. For a matrix $A \in M_{m \times n}(S)$, let the $i$ th row sum and $j$ th column sum be denoted by $r_{i}(A)$ and 
$c_{j}(A)$ respectively. Now, if we concentrate on the weighted sum $\mathcal{S}:=\sum_{A \in M_{m \times n}(S)}\left(\prod_{i=1}^{m} r_{i}(A)\right)\left(\prod_{j=1}^{n} c_{j}(A)\right)$, a bare hand calculation yields that the sum is 34 , if we take $m=n=2$ and $q=1$. But even for these small values of $m, n, q$, one has to check as many as 16 cases to get $\mathcal{S}$. The difficulty arises quickly if we increase $m, n, q$. For instance, if we choose $m=4, n=5, q=1$, then one has to check $2^{20}=1048576$ cases (in this case, Theorem 1.5 answers the sum to be 695664640). Choosing $q=3$ under the same setup (i.e. $m=4$ and $n=5$ ), gives rise to a checking of $4^{20}$ cases and the difficulty keeps increasing. In this paper, we have found explicit formulae for $\mathcal{S}$. This enumeration also gives rise to a few interesting identities satisfying nice reciprocity phenomena.

Throughout this paper, we will be using a few notations defined below. We shall use the notation $p(m)$ to denote the set of all partitions of $m$. If $\sigma$ is a partition of $m$, then the number of $i$ 's occurring in $\sigma$ is denoted by $\sigma_{i}$. For example, suppose $\sigma=3+2+2+1$ is a partition of 8 , then $\sigma_{1}=1, \sigma_{2}=2, \sigma_{3}=1$ and $\sigma_{4}, \sigma_{5}, \cdots, \sigma_{8}=0$. Thus $\sum_{i=1}^{m} i \sigma_{i}=m$. Define $|\sigma|:=\sum_{i=1}^{m} \sigma_{i}$. The minimum of two integers $m$ and $n$ will be denoted by $m \wedge n$. Let $S$ be a set. Then the $n$-fold cartesian product $S \times S \times \cdots \times S$ (n times) is denoted by $S^{n}$. For $z \in \mathbb{C}, \operatorname{Re}(z)$ will denote the real part of $z$.

Now, we are in a position to state our results. Our first result will be on the enumeration of $m \times n$ matrices with entries from the set $S:=\{0,1, \ldots, q\}$ such that the row sums and the column sums are divisible by a given integer $k$. Let $M(m, n, q, k)$ denote the number of matrices in $M_{m \times n}(S)$ with row sums and column sums divisible by $k$. Then we have the following.

Theorem 1.1.

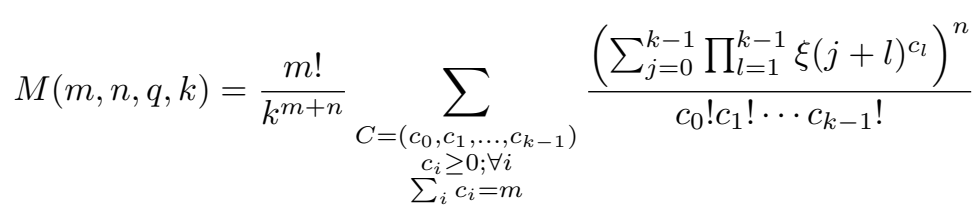

where, $\xi_{k}$ is a primitive $k$ th root of unity and $\xi(x):=\sum_{t=0}^{q} \xi_{k}^{x t}$.

In the above case, if we concentrate only on the $n \times n$ symmetric matrices, then we obtain our next theorem. Let $H(n, q, k)$ denote the number of $n \times n$ symmetric matrices having entries from $S$ (defined previously) with row sums and column sums divisible by $k$. Then we have the following.

Theorem 1.2.

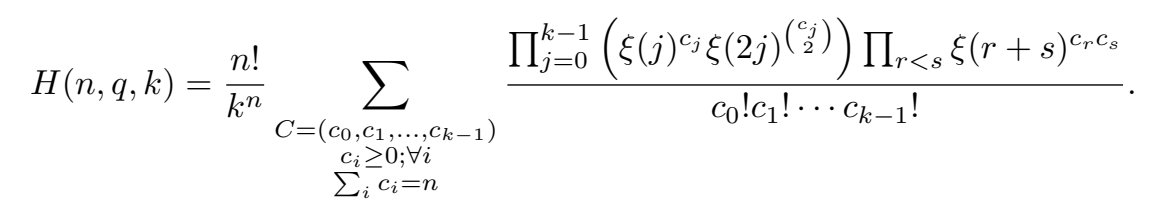

Now, in order to enumerate labelled regular graphs, we have to concentrate on traceless $(0,1), n \times n$ symmetric matrices. By a slight modification in the above setup and considering $q=1$, we can in fact write down a $\xi_{k}$ free expression which will help us enumerating labelled regular graphs. Our next theorem is on that. It says, if $\mathfrak{F}(n, k)$ denotes the set of traceless $(0,1), n \times n$ symmetric matrices with each line sum divisible by $k$ and $F(n, k):=|\mathfrak{F}(n, k)|$, then we have the following,

Theorem 1.3.

$$
F(n, k)=\frac{n ! 2^{\left(\begin{array}{c}
n \\
2
\end{array}\right)}}{k^{n}} \sum_{\substack{C=\left(c_{0}, c_{1}, \ldots, c_{k-1}\right) \\
c_{i} \geq 0 ; \forall i \\
\sum_{i} c_{i}=n}} \frac{\mathcal{C}(\mathfrak{C}(C))\left(\prod_{j=0}^{k-1} \mathcal{C}(2 j)^{\left(\begin{array}{c}
c_{j} \\
2
\end{array}\right)} \prod_{r<s} \mathcal{C}(r+s)^{c_{r} c_{s}}\right)}{c_{0} ! c_{1} ! \cdots c_{k-1} !}
$$

where $\mathcal{C}(x):=\cos \left(\frac{x \pi}{k}\right)$ and $\mathfrak{C}(C):=(n-1)\left(\sum_{j=0}^{k-1} j c_{j}\right)$ for any $k$-tuple $C=\left(c_{0}, c_{1}, \ldots, c_{k-1}\right)$ where $c_{i} \geq 0$ for all $i$ and $\sum_{i} c_{i}=n$.

Now, as a consequence of Theorem 1.3, we can find an explicit formula for counting labeled $k$-regular graphs with $n$ vertices. Suppose $R(n, k)$ denotes the number of labeled $k$-regular graphs with $n$ vertices. Then we have the following.

Theorem 1.4.

$$
R(n, k)= \begin{cases}\sum_{i=0}^{n}(-1)^{i}\left(\begin{array}{c}
n \\
i
\end{array}\right) F(n-i, k), & k>\frac{n-1}{2}, \\
F(n, k)+2 \sum_{i=1}^{n}(-1)^{i}\left(\begin{array}{c}
n \\
i
\end{array}\right) F(n-i, k), & k=\frac{n-1}{2} .\end{cases}
$$

When $k<\frac{n-1}{2}, R(n, k)$ can be evaluated from the above theorem using the trivial relation $R(n, k)=R(n, n-$ $k-1)$. 
Let us now come to the weighted enumeration of rectangular matrices, as mentioned earlier. Let us denote the given set of integers $\{0,1, \ldots, q\}$ by $S$. Let $M_{m \times n}(S)$ denote the set of all $m \times n$ matrix with entries from the set $S:=\{0,1, \ldots, q\}$. For a matrix $A \in M_{m \times n}(S)$, let the $i$ th row sum and $j$ th column sum be denoted by $r_{i}(A)$ and $c_{j}(A)$ respectively. Then we have the following,

\section{Theorem 1.5.}

$$
\begin{aligned}
\sum_{A \in M_{m \times n}(S)}\left(\prod_{i=1}^{m} r_{i}(A)\right)\left(\prod_{j=1}^{n} c_{j}(A)\right) & =\frac{m ! n !(q+1)^{m n} q^{(m+n)}}{2^{(m+n)}} \sum_{\substack{\sigma \in p(m),|\sigma| \leq m \wedge n}} \frac{A(m, n, \sigma)}{q^{|\sigma|}} \prod_{r=1}^{m}\left(\frac{q(3 m+r)+2 r}{r !}\right)^{\sigma_{r}} \\
& \left.=\frac{m ! n !(q+1)^{m n} q^{(m+n)}}{2^{(m+n)}} \sum_{\substack{\pi \in p(n) \\
|\pi| \leq m \wedge n}} \frac{A(n, m, \pi)}{q^{|\pi|}} \prod_{t=1}^{n}\left(\frac{q(3 n+t)+2 t}{t !}\right)^{\pi_{t}}\right) \\
& =\frac{q^{(m+n)}(q+1)^{m n} m^{n} n^{m}}{2^{(m+n)}} \sum_{i=0}^{m \wedge n} i !\left(\begin{array}{c}
m \\
i
\end{array}\right)\left(\begin{array}{c}
n \\
i
\end{array}\right)\left(\frac{q+2}{3 m n q}\right)^{i},
\end{aligned}
$$

where $A(x, y, \lambda):=\frac{x^{(y-|\lambda|)}}{\lambda_{1} ! \lambda_{2} ! \cdots \lambda_{x} !(y-|\lambda|) ! 3|\lambda|}$ for non-negative integers $x, y$ and $\lambda \in p(x)$.

Theorem 1.5, in its full generality exhibits a nice reciprocity phenomenon. In particular, letting $q=1$, the above theorem specializes to the following nice identity.

Corollary 1.1.

$$
\begin{aligned}
\sum_{\substack{\sigma \in p(m),|\sigma| \leq m \wedge n}} \frac{m^{(n-|\sigma|)}}{\sigma_{1} ! \sigma_{2} ! \cdots \sigma_{m} !(n-|\sigma|) !} \prod_{r=1}^{m}\left(\frac{m+r}{r !}\right)^{\sigma_{r}} & =\sum_{\substack{\pi \in p(n),|\pi| \leq m \wedge n}} \frac{n^{(m-|\pi|)}}{\pi_{1} ! \pi_{2} ! \cdots \pi_{n} !(m-|\pi|) !} \prod_{t=1}^{n}\left(\frac{n+t}{t !}\right)^{\pi_{t}} \\
& =\frac{m^{n} n^{m}}{m ! n !} \sum_{i=0}^{m \wedge n}\left(\begin{array}{c}
m \\
i
\end{array}\right)\left(\begin{array}{c}
n \\
i
\end{array}\right) \frac{i !}{(m n)^{i}} .
\end{aligned}
$$

\section{Proofs of the results}

Proof of Theorem 1.1. Before getting into the proof, let us make a quick observation first.

Observation 1: Let $\mathcal{P}\left(x_{1}, x_{2}, \ldots, x_{s}\right)$ be a polynomial in $\mathbb{C}\left[x_{1}, x_{2}, \ldots, x_{s}\right]$ for some $s \in \mathbb{N}$ and $a \in \mathbb{N}$ be any given positive integer. Suppose we are interested in finding the sum of the coefficients of the monomials in which every variable $x_{i}$ has exponent divisible by $a$. Let us denote this required sum by $S(\mathcal{P}, a)$. To face the above scenario, let us consider the set $\mathcal{R}_{a}:=\left\{\xi_{a}^{0}, \xi_{a}^{1}, \ldots, \xi_{a}^{(a-1)}\right\}$, where $\xi_{a}$ denotes a primitive $a$ th root of unity in $\mathbb{C}$.

Let us define a map $\mathcal{D}: \mathbb{C}\left[x_{1}, x_{2}, \ldots, x_{n}\right] \rightarrow \mathbb{C}\left[x_{1}, x_{2}, \ldots, x_{n-1}\right]$ for all $n \in \mathbb{N}$, as follows,

$$
\mathcal{D}\left(f\left(x_{1}, x_{2}, \ldots, x_{n}\right)\right):=\frac{1}{a} \sum_{z \in \mathcal{R}_{a}} f\left(x_{1}, x_{2}, \ldots, x_{n-1}, z\right) .
$$

It is easy to see that if

$$
f=\sum_{\left(m_{1}, m_{2}, \ldots, m_{n}\right) \in(\mathbb{N} \cup\{0\})^{n}} c_{m_{1}, m_{2}, \ldots, m_{n}} x_{1}^{m_{1}} x_{2}^{m_{2}} \cdots x_{n}^{m_{n}}
$$

then,

$$
\mathcal{D}(f)=\sum_{\left(m_{1}, m_{2}, \ldots, m_{n}\right) \in(\mathbb{N} \cup\{0\})^{n}} \bar{c}_{m_{1}, m_{2}, \ldots, m_{n}} x_{1}^{m_{1}} x_{2}^{m_{2}} \cdots x_{n-1}^{m_{n-1}}
$$

where $\bar{c}_{m_{1}, m_{2}, \ldots, m_{n}}=c_{m_{1}, m_{2}, \ldots, m_{n}}$ when $m_{n}$ is divisible by $a$ and $\bar{c}_{m_{1}, m_{2}, \ldots, m_{n}}=0$ otherwise. So, it is also easy to see that $S(\mathcal{P}, a)=\mathcal{D}^{s}(\mathcal{P})$, where $\mathcal{D}^{s}(\mathcal{P})$ means $\mathcal{D}$ acts iteratively $s$-times over $\mathcal{P}$. On the other hand, from the definition of $\mathcal{D}$ it is clear that $\mathcal{D}^{t}(\mathcal{P})=\frac{1}{a^{t}} \sum_{z \in \mathcal{R}_{a}^{t}} \mathcal{P}\left(x_{1}, x_{2}, \ldots, x_{s-t}, z\right)$ for all $0 \leq t \leq s$. So, it follows that,

$$
S(\mathcal{P}, a)=\mathcal{D}^{s}(\mathcal{P})=\frac{1}{k^{s}} \sum_{z \in \mathcal{R}_{a}^{s}} \mathcal{P}(z) .
$$

Now consider the polynomial

$$
P\left(x_{1}, x_{2}, \ldots, x_{m}, y_{1}, y_{2}, \ldots, y_{n}\right):=\prod_{i=1}^{m} \prod_{j=1}^{n}\left(\sum_{t=0}^{q}\left(x_{i} y_{j}\right)^{t}\right) .
$$


In order to find $M(m, n, q, k)$, we have to concentrate on the set $\mathcal{R}_{k}$ (defined in observation 1). With a slight abuse of notation, we will proceed considering $\mathcal{R}$ to be same as $\mathcal{R}_{k}$, unless otherwise mentioned. Now, in light of observation 1, one can see that

$$
M(m, n, q, k)=\frac{1}{k^{m+n}} \sum_{\mathbf{Z} \in \mathcal{R}^{m+n}} P(\mathbf{Z}) .
$$

Again, it is quite clear that

$$
\frac{1}{k^{m+n}} \sum_{\mathbf{Z} \in \mathcal{R}^{m+n}} P(\mathbf{Z})=\frac{1}{k^{m+n}} \sum_{\substack{\mathbf{Z}_{m} \in \mathcal{R}^{m} \\ \mathbf{Z}_{n} \in \mathcal{R}^{n}}} P\left(\mathbf{Z}_{m}, \mathbf{Z}_{n}\right) .
$$

So, first let us try to compute $\sum_{\mathbf{z}_{n} \in \mathcal{R}^{n}} P\left(X, \mathbf{z}_{n}\right)$, where $X=\left(x_{1}, x_{2}, \ldots, x_{m}\right)$. Now, for a particular $\mathbf{Z}_{n}=$ $\left(z_{1}, z_{2}, \ldots, z_{n}\right) \in \mathcal{R}^{n}$

$$
P\left(X, \mathbf{Z}_{n}\right)=\prod_{i=1}^{m} \prod_{j=1}^{n}\left(\sum_{t=0}^{q}\left(x_{i} z_{j}\right)^{t}\right) .
$$

So,

$$
\sum_{\mathbf{z}_{n} \in \mathcal{R}^{n}} P\left(X, \mathbf{Z}_{n}\right)=\left(\sum_{z \in \mathcal{R}} \prod_{i=1}^{m}\left(\sum_{t=0}^{q}\left(x_{i} z\right)^{t}\right)\right)^{n} .
$$

Hence,

$$
\sum_{\mathbf{Z}_{m} \in \mathcal{R}^{m}} P\left(\mathbf{Z}_{m}, \mathbf{Z}_{n}\right)=\sum_{\mathbf{Z}_{m} \in \mathcal{R}^{m}}\left(\sum_{z \in \mathcal{R}} \prod_{i=1}^{m}\left(\sum_{t=0}^{q}\left(\mathfrak{z}_{i} z\right)^{t}\right)\right)^{n}
$$

where, $\mathbf{Z}_{m}=\left(\mathfrak{z}_{1}, \mathfrak{z}_{2}, \ldots, \mathfrak{z}_{m}\right) \in \mathcal{R}^{m}$. So, for a particular $\mathbf{Z}_{m}=\left(\mathfrak{z}_{1}, \mathfrak{z}_{2}, \ldots, \mathfrak{z}_{m}\right) \in \mathcal{R}^{m}$, if $\xi_{k}^{i}$ occurs $c_{i}$ times in the multiset $\left\{\mathfrak{z}_{1}, \mathfrak{z}_{2}, \ldots, \mathfrak{z}_{m}\right\}$, then we have the following,

$$
\begin{aligned}
\sum_{\substack{\mathbf{Z}_{m} \in \mathcal{R}^{m} \\
\mathbf{Z}_{n} \in \mathcal{R}^{n}}} P\left(\mathbf{Z}_{m}, \mathbf{Z}_{n}\right) & =\sum_{\mathbf{z}_{m}=\left(\begin{array}{l}
\left.\mathfrak{z}_{1}, \mathfrak{z}_{2}, \ldots, \mathfrak{z}_{m}\right) \in \mathcal{R}^{m} \\
\end{array}\right.}\left(\sum_{z \in \mathcal{R}} \prod_{i=1}^{m}\left(\sum_{t=0}^{q}\left(\mathfrak{z}_{i} z\right)^{t}\right)\right)^{n} \\
& \sum_{\substack{\left(c_{0}, c_{1}, \ldots, c_{k-1}\right) \\
c_{l} \geq 0 ; \forall l l \\
\sum_{l} c_{l}=m}} \frac{m !}{c_{0} ! c_{1} ! \cdots c_{k-1} !}\left(\sum_{j=0}^{k-1} \prod_{l=1}^{k-1}\left(\sum_{t=0}^{q}\left(\xi_{k}^{(j+l)}\right)^{t}\right)^{c_{l}}\right)^{n} .
\end{aligned}
$$

Remark: Theorem 1.1 can be directly used to answer the following question raised in the introduction. What is the number of $m \times n$ matrices with entries from the set $\{0,1, \ldots, q\}$ such that all the row sums and column sums are even integers, if $q$ is even? The answer turns out to be

$$
\frac{1}{2^{m+n}} \sum_{t=0}^{n}\left(\begin{array}{c}
n \\
t
\end{array}\right)\left((q+1)^{t}+(q+1)^{n-t}\right)^{m}=\frac{1}{2^{m+n}} \sum_{t=0}^{m}\left(\begin{array}{c}
m \\
t
\end{array}\right)\left((q+1)^{t}+(q+1)^{m-t}\right)^{n} .
$$

Proof of Theorem 1.3. Consider the polynomial

$$
G\left(x_{1}, x_{2}, \ldots, x_{n}\right):=\prod_{1 \leq i<j \leq n}\left(1+x_{i} x_{j}\right) .
$$

Let $\mathcal{R}:=\left\{\xi_{k}^{0}, \xi_{k}^{1}, \ldots, \xi_{k}^{(k-1)}\right\}$, where $\xi_{k}$ denotes a primitive $k$-th root of unity. Then again observation 1 enlightens us to see that

$$
F(n, k)=\frac{1}{k^{n}} \sum_{\mathbf{Z} \in \mathcal{R}^{n}} G(\mathbf{Z}) .
$$

For a particular $\mathbf{Z}=\left(z_{1}, z_{2}, \ldots, z_{n}\right) \in \mathcal{R}^{n}$, suppose the number of occurrences of $\xi_{k}^{i}$ in the multiset $\left\{z_{1}, z_{2}, \ldots, z_{n}\right\}$ be $c_{i}$. Then

$$
G(\mathbf{Z})=\prod_{j=0}^{k-1}\left(1+\xi_{k}^{2 j}\right)^{\left(\begin{array}{c}
c_{j} \\
2
\end{array}\right)} \prod_{r<s}\left(1+\xi_{k}^{(r+s)}\right)^{c_{r} c_{s}} .
$$

So we have

$$
F(n, k)=\frac{1}{k^{n}} \sum_{\substack{\left(c_{0}, c_{1}, \ldots, c_{k-1}\right) \\
c_{i} \geq 0 ; \forall i v i \\
\sum_{i} c_{i}=n}} \frac{n !}{c_{0} ! c_{1} ! \cdots c_{k-1} !} \prod_{j=0}^{k-1}\left(1+\xi_{k}^{2 j}\right)^{\left(\begin{array}{c}
c_{j} \\
2
\end{array}\right)} \prod_{r<s}\left(1+\xi_{k}^{(r+s)}\right)^{c_{r} c_{s}}
$$




$$
=\operatorname{Re}\left(\frac{1}{k^{n}} \sum_{\substack{\left(c_{0}, c_{1}, \ldots, c_{k-1}\right) \\ c_{i} \geq 0 ; \forall i \\ \sum_{i} c_{i}=n}} \frac{n !}{c_{0} ! c_{1} ! \cdots c_{k-1} !} \prod_{j=0}^{k-1}\left(1+\xi_{k}^{2 j}\right)^{\left({ }^{c_{j}}\right)} \prod_{r<s}\left(1+\xi_{k}^{(r+s)}\right)^{c_{r} c_{s}}\right)
$$

(since $F(n, k)$ is real)

$$
\begin{aligned}
& =\frac{1}{k^{n}} \sum_{\substack{\left(c_{0}, c_{1}, \ldots, c_{k-1}\right) \\
c_{i} \geq 0, \forall i \\
\sum_{i} c_{i}=n}} \frac{n !}{c_{0} ! c_{1} ! \cdots c_{k-1} !} \operatorname{Re}\left(\prod_{j=0}^{k-1}\left(1+\xi_{k}^{2 j}\right)^{\left(\begin{array}{c}
c_{j} \\
2
\end{array}\right)} \prod_{r<s}\left(1+\xi_{k}^{(r+s)}\right)^{c_{r} c_{s}}\right)
\end{aligned}
$$

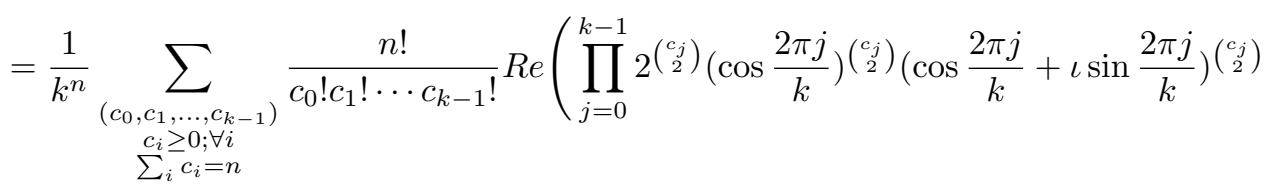

$$
\begin{aligned}
& \left.\times \prod_{r<s} 2^{c_{r} c_{s}}\left(\cos \frac{\pi(r+s)}{k}\right)^{c_{r} c_{s}}\left(\cos \frac{\pi(r+s)}{k}+\iota \sin \frac{\pi(r+s)}{k}\right)^{c_{r} c_{s}}\right) \\
& =\frac{\left.n ! 22^{(} \begin{array}{c}
n \\
2
\end{array}\right)}{k^{n}} \sum_{\substack{\left(c_{0}, c_{1}, \ldots, c_{k-1}\right) \\
c_{i} \geq 0 ; \forall i \\
\sum_{i} c_{i}=n}} \frac{\cos \left(\left(\sum_{j=0}^{k-1} 2 j\left(\begin{array}{c}
c_{j} \\
2
\end{array}\right)+\sum_{r<s}(r+s) c_{r} c_{s}\right) \frac{\pi}{k}\right)}{c_{0} ! c_{1} ! \cdots c_{k-1} !} \\
& \times\left(\prod_{j=0}^{k-1}\left(\cos \frac{2 \pi j}{k}\right)^{\left(\begin{array}{c}
c_{j} \\
2
\end{array}\right)} \prod_{r<s}\left(\cos \frac{\pi(r+s)}{k}\right)^{c_{r} c_{s}}\right) .
\end{aligned}
$$

We can simplify the above relation using the following simple identity

$$
\sum_{j=0}^{k-1} 2 j\left(\begin{array}{c}
c_{j} \\
2
\end{array}\right)+\sum_{r<s}(r+s) c_{r} c_{s}=(n-1) \sum_{j=0}^{k-1} j c_{j},
$$

for all $k$-tuples $\left(c_{0}, c_{1}, \ldots, c_{k-1}\right)$ satisfying $c_{i} \geq 0$ for all $i$ and $\sum_{i} c_{i}=n$. To prove this identity, take a particular $\left(c_{0}, c_{1}, \ldots, c_{k-1}\right) \in(\mathbb{N} \cup\{0\})^{k}$ satisfying $\sum_{i} c_{i}=n$. Now consider the polynomial $p(x)$ in $\mathbb{Z}[x]$, defined as follows

$$
p(x):=\left(\sum_{j} c_{j} x^{j}\right)^{2}-\left(\sum_{j} c_{j} x^{2 j}\right)
$$

One can easily see that

$$
\left(\sum_{j} c_{j} x^{j}\right)^{2}-\left(\sum_{j} c_{j} x^{2 j}\right)=\sum_{j}\left(c_{j}^{2}-c_{j}\right) x^{2 j}+2 \sum_{r<s} c_{r} c_{s} x^{r+s}
$$

Taking the derivative with respect to $x$ on both sides at $x=1$ and dividing both sides by 2 and using the condition $\sum_{i} c_{i}=n$, one gets the desired relation.

In the light of the above identity and all the previous calculations, one can get

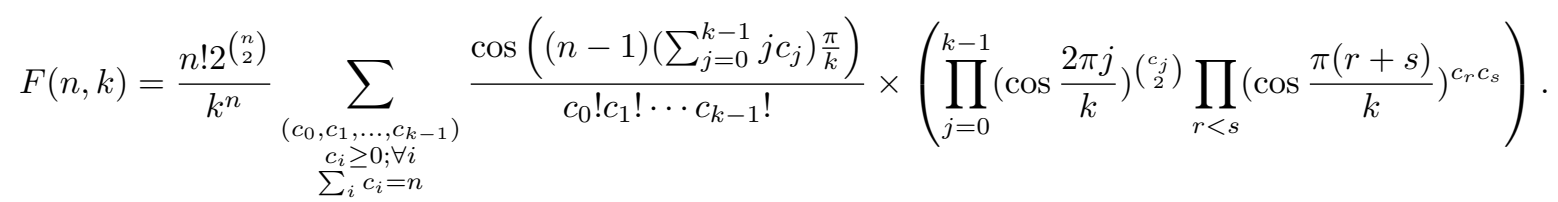

A class of identities can be deduced from the Theorem 1.3. For instance, the following nice identity is an immediate corollary of the above.

Corollary 2.1.

$$
2=\frac{n ! 2^{\left(\begin{array}{c}
n \\
2
\end{array}\right)}}{(n-1)^{n}} \sum_{\substack{\left(c_{0}, c_{1}, \ldots, c_{n-2}\right) \\
c_{i} \geq 0 ; \forall i \\
\sum_{i} c_{i}=n}} \frac{(-1)^{\left(\sum_{j=0}^{n-2} j c_{j}\right)}}{c_{0} ! c_{1} ! \cdots c_{n-2} !}\left(\prod_{j=0}^{n-2}\left(\cos \frac{2 \pi j}{n-1}\right)^{\left(\begin{array}{c}
c_{j} \\
2
\end{array}\right)} \prod_{r<s}\left(\cos \frac{\pi(r+s)}{n-1}\right)^{c_{r} c_{s}}\right)
$$


Proof. Putting $k=n-1$ in Theorem 1.3, yields the result, since the following two are the only matrices in $\mathfrak{F}(n, n-1)$,

$$
\left(\begin{array}{cccc}
0 & 0 & \cdots & 0 \\
0 & 0 & \cdots & 0 \\
\vdots & \vdots & \ddots & \vdots \\
0 & 0 & \cdots & 0
\end{array}\right),\left(\begin{array}{cccc}
0 & 1 & \cdots & 1 \\
1 & 0 & \cdots & 1 \\
\vdots & \vdots & \ddots & \vdots \\
1 & 1 & \cdots & 0
\end{array}\right)
$$

Using similar techniques, used in the proof of Theorems 1.1 and 1.3, we obtain Theorem 1.2. So the details are omitted.

Proof of Theorem 1.4. With Theorem 1.3 in hand, we are in a state to count labelled $k$-regular graphs in $n$ vertices. Theorem 1.3 gives us the number of traceless $n \times n,(0,1)$ symmetric matrices with line sums divisible by $k$. Now, one can see that, for a traceless $n \times n,(0,1)$ matrix, the maximum possible line sum is $n-1$. So if we choose $k$ to be greater than $\frac{n-1}{2}$, the only possible line sums, divisible by $k$ are 0 and $k$ itself. So, in order to find $R(n, k)$, we have to eliminate the matrices having at least one row (equivalently column) sum zero from $\mathfrak{F}(n, k)$. Using the principle of inclusion and exclusion, we get the following

$$
R(n, k)=\sum_{i=0}^{n}(-1)^{i}\left(\begin{array}{c}
n \\
i
\end{array}\right) F(n-i, k) .
$$

When $k=\frac{n-1}{2}$, it is not difficult to see that $\mathfrak{F}(n, k)$ consists of precisely three types of matrices, Type $A$, Type $B$ and Type $C$, say, where Type $A$ consists of the matrices with at least one line sum 0 , Type $B$ consists of matrices with at least one line sum $2 k$ and Type $C$ consists of matrices with line sums precisely equal to $k$. Now the number of Type $A$ matrices is $\sum_{i=1}^{n}(-1)^{i+1}\left(\begin{array}{c}n \\ i\end{array}\right) F(n-i, k)$. Also it is easy to see that Type A and Type B matrices are equinumerous. So, by the principle of inclusion and exclusion, we have

$$
R(n, k)=F(n, k)+2\left(\sum_{i=1}^{n}(-1)^{i}\left(\begin{array}{l}
n \\
i
\end{array}\right) F(n-i, k)\right) .
$$

Proof of Theorem 1.5. The proof of this theorem is divided into two parts. The first part is devoted to proving the first two equalities of Theorem 1.5 and the second part is devoted to proving the last equality of the same theorem. The main task in the whole proof is to evaluate an iterated partial derivative. In the first part of the proof, we have used Faà di Bruno's formula[ [8] proposition 1] and in the second part, we have used the partial differencial operator technique to evaluate the iterated partial derivative.

Proof of the first part: Recall that we have defined

$$
\mathcal{S}=\sum_{A \in M_{m \times n}(S)}\left(\prod_{i=1}^{m} r_{i}(A)\right)\left(\prod_{j=1}^{n} c_{j}(A)\right)
$$

in the introduction. Now consider the polynomial

$$
P\left(x_{1}, x_{2}, \ldots, x_{m}, y_{1}, y_{2}, \ldots, y_{n}\right):=\prod_{i=1}^{m} \prod_{j=1}^{n}\left(\sum_{t=0}^{q}\left(x_{i} y_{j}\right)^{t}\right) .
$$

Then one can see that

$$
\mathcal{S}=\frac{\partial^{m+n}}{\partial x_{1} \partial x_{2} \cdots \partial x_{m} \partial y_{1} \partial y_{2} \cdots \partial y_{n}} P\left(x_{1}, x_{2}, \ldots, x_{m}, y_{1}, y_{2}, \ldots, y_{n}\right)
$$

evaluated at $x_{i}=1=y_{j}$ for all $1 \leq i \leq m, 1 \leq j \leq n$.

Let $Q\left(x_{1}, x_{2}, \ldots, x_{m}\right):=\frac{\partial^{n}}{\partial y_{1} \partial y_{2} \cdots \partial y_{n}} P\left(x_{1}, x_{2}, \ldots, x_{m}, y_{1}, y_{2}, \ldots, y_{n}\right)$ evaluated at $y_{1}=y_{2}=\cdots=y_{n}=1$.

Now, $P\left(x_{1}, x_{2}, \ldots, x_{m}, y_{1}, y_{2}, \ldots, y_{n}\right)$ can be written as

$$
P\left(x_{1}, x_{2}, \ldots, x_{m}, y_{1}, y_{2}, \ldots, y_{n}\right)=\prod_{j=1}^{n}\left(\sum_{k=0}^{m q} \widetilde{h_{k}}(\bar{X}) y_{j}^{k}\right)
$$


where, $\widetilde{h_{k}}(\bar{X}):=\sum_{\psi \in \beta_{m}(k)} X^{\psi}, \beta_{m}(k)$ is the set of all ordered tuples of integers $\psi=\left(b_{1}, b_{2}, \ldots, b_{m}\right)$ such that $b_{1}+b_{2}+\cdots+b_{m}=k$ with $0 \leq b_{i} \leq q$ for all $i=1,2, \ldots, m$ and $X^{\psi}=x_{1}^{b_{1}} x_{2}^{b_{2}} \cdots x_{m}^{b_{m}}$. We call $\widetilde{h_{k}}(\bar{X})$ as restricted homogeneous symmetric function of degree $k$ in $m$ variables.

So, $Q\left(x_{1}, x_{2}, \ldots, x_{m}\right)$ becomes $\left(\sum_{k=0}^{m q} k \widetilde{h_{k}}(\bar{X})\right)^{n}$.

Thus we have $\mathcal{S}=\frac{\partial^{m}}{\partial x_{1} \partial x_{2} \cdots \partial x_{m}} Q\left(x_{1}, x_{2}, \ldots, x_{m}\right)$, evaluated at $x_{1}=x_{2}=\cdots=x_{m}=1$.

Let $z=\sum_{k=0}^{m q} k \widetilde{h_{k}}(\bar{X})$ and $f(z)=z^{n}\left(=Q\left(x_{1}, x_{2}, \ldots, x_{m}\right)\right)$.

We have to evaluate $\frac{\partial^{m} f(z)}{\partial x_{1} \partial x_{2} \cdots \partial x_{m}}$ at $x_{1}=x_{2}=\cdots=x_{m}=1$. We shall use the following generalized version of Faà di Bruno's formula[ [8] proposition 1] to accomplish this task:

$$
\frac{\partial^{m} f(z)}{\partial x_{1} \partial x_{2} \cdots \partial x_{m}}=\sum_{\eta} f^{|\eta|}(z) \prod_{B \in \eta} \frac{\partial^{|B|} z}{\prod_{i \in B} \partial x_{i}}
$$

where the summation runs over all partitions $\eta$ of the set $\{1,2, \ldots, m\}$ (not to be confused with the integer partitions, previously dealt with) and the product is over all of the parts (or blocks) $B$ of the partition $\eta$ and we denote the number of members of any set $T$ by $|T|$ (the same notation has already occurred previously, but in a different context. So when the context is clear, no confusion should arise). Note that $f^{|\eta|}(z)=\frac{n !}{(n-|\eta|) !} z^{(n-|\eta|)}$, when $|\eta| \leq n ; f^{|\eta|}(z)=0$, otherwise. So, the only set partitions $\eta$ with $|\eta| \leq n$ will be our matter of interest.

Now, for a particular set partition $\eta$ of the set $\{1,2, \ldots, m\}$, let us denote the product, $\prod_{B \in \eta} \frac{\partial^{|B|} z}{\prod_{i \in B} \partial x_{i}}$ evaluated at $x_{1}=x_{2}=\cdots=x_{m}=1$, by $\partial(\eta)$.

Since, $\widetilde{h_{k}}(\bar{X})$ is symmetric, $z$ is also symmetric. So the value of $\frac{\partial^{|B|} z}{\prod_{i \in B} \partial x_{i}}$ when evaluated at $x_{1}=x_{2}=\cdots=$ $x_{m}=1$ does not depend on the choice of the elements in $B$ but only on $|B|$. So if $|B|=l$ it suffices to evaluate $\frac{\partial^{l} z}{\partial x_{1} \partial x_{2} \cdots \partial x_{l}}$ at $x_{1}=x_{2}=\cdots=x_{m}=1$. Now

$$
\begin{aligned}
\frac{\partial^{l} z}{\partial x_{1} \partial x_{2} \cdots \partial x_{l}} & =\frac{\partial^{l}}{\partial x_{1} \partial x_{2} \cdots \partial x_{l}} \sum_{k=0}^{m q} k \widetilde{h_{k}}(\bar{X}) \\
& =\sum_{k=0}^{m q} \frac{\partial^{l}}{\partial x_{1} \partial x_{2} \cdots \partial x_{l}}\left(\sum_{\psi \in \beta_{m}(k)} X^{\psi}\right)=\sum_{k=0}^{m q} k\left(\sum_{\psi=\left(b_{1}, \ldots, b_{m}\right) \in \beta_{m}(k)} b_{1} b_{2} \cdots b_{l}\right)
\end{aligned}
$$

(after evaluating the partial derivative at $x_{1}=x_{2}=\cdots=x_{m}=1$ ).

Now, let us simplify the summation above in a different manner. Let us define the polynomial

$$
R\left(x_{1}, x_{2}, \ldots, x_{m}, t\right):=\sum_{i=1}^{m}\left(1+x_{i} t+x_{i}^{2} t^{2}+\cdots+x_{i}^{q} t^{q}\right) .
$$

So, it evidently follows that

$$
\sum_{k=0}^{m q} k\left(\sum_{\psi=\left(b_{1}, \ldots, b_{m}\right) \in \beta_{m}(k)} b_{1} b_{2} \cdots b_{l}\right)=\frac{\partial^{l+1}}{\partial t \partial x_{1} \partial x_{2} \cdots \partial x_{l}} R\left(x_{1}, x_{2}, \ldots, x_{m}, t\right)
$$

evaluated at $x_{1}=x_{2}=\cdots=x_{m}=t=1$.

Now

$$
\frac{\partial^{l} R}{\partial x_{1} \partial x_{2} \cdots \partial x_{l}}=\prod_{i=1}^{l} \frac{\partial}{\partial x_{i}}\left(\sum_{p=0}^{q}\left(x_{i} t\right)^{p}\right) \prod_{i=l+1}^{m}\left(\sum_{p=0}^{q}\left(x_{i} t\right)^{p}\right) .
$$

Evaluating the above derivative at $x_{1}=x_{2}=\cdots=x_{m}=1$ we get, $\left(\sum_{p=1}^{q} p t^{p}\right)^{l}\left(\sum_{p=0}^{q} t^{p}\right)^{(m-l)}$. Hence, we have $\frac{\partial^{l+1}}{\partial t \partial x_{1} \partial x_{2} \cdots \partial x_{l}} R\left(x_{1}, x_{2}, \ldots, x_{m}, t\right)$ evaluated at $x_{1}=x_{2}=\cdots=x_{m}=t=1$ is same as $\frac{d}{d t}\left(\sum_{p=1}^{q} p t^{p}\right)^{l}\left(\sum_{p=0}^{q} t^{p}\right)^{(m-l)}$ evaluated at $t=1$.

Now

$\frac{d}{d t}\left(\sum_{p=1}^{q} p t^{p}\right)^{l}\left(\sum_{p=0}^{q} t^{p}\right)^{(m-l)}=l\left(\sum_{p=1}^{q} p t^{p}\right)^{(l-1)}\left(\sum_{p=1}^{q} p^{2} t^{(p-1)}\left(\sum_{p=0}^{q} t^{p}\right)^{(m-l)}+(m-l)\left(\sum_{p=1}^{q} p t^{p}\right)^{l}\left(\sum_{p=0}^{q} t^{p}\right)^{(m-l-1)}\left(\sum_{p=1}^{q} p t^{(p-1)}\right)\right.$.

Evaluating the above derivative at $t=1$, we get

$$
l\left(\frac{q(q+1)}{2}\right)^{(l-1)}\left(\frac{q(q+1)(2 q+1)}{6}\right)(q+1)^{(m-l)}+(m-l)\left(\frac{q(q+1)}{2}\right)^{(l+1)}(q+1)^{(m-l-1)}
$$




$$
=\frac{q^{l}(q+1)^{m}}{2^{(l+1)}}\left(\frac{q(l+3 m)+2 l}{3}\right) .
$$

So, finally we have the following

$$
\frac{\partial^{l} z}{\partial x_{1} \partial x_{2} \cdots \partial x_{l}}=\frac{q^{l}(q+1)^{m}}{2^{(l+1)}}\left(\frac{q(l+3 m)+2 l}{3}\right) .
$$

when evaluated at $x_{1}=x_{2}=\cdots=x_{m}=1$. Note that, if we put $l=0$ in the above expression, we get

$$
z=\frac{m q}{2}(q+1)^{m}
$$

when evaluated at $x_{1}=x_{2}=\cdots=x_{m}=1$.

As we have already mentioned, if $\zeta(\in p(m))$ has $l_{1}, l_{2}, \ldots, l_{d}$ as parts and $\eta=\left\{B_{1}, B_{2}, \ldots, B_{d}\right\}$ and $\dot{\eta}=$ $\left\{B_{1}, B_{2}, \ldots, B_{d}\right\}$ are any two set partitions of $\{1,2, \ldots, m\}$ with $\left|B_{i}\right|=\left|B_{i}\right|=l_{i}$ for all $i=1,2, \ldots, d$, then $\partial(\eta)=\partial(\eta)$. So, for any particular partition $\sigma$ of $m$, there will be $\frac{m !}{\sigma_{1} ! \sigma_{2} ! \cdots \sigma_{m} !(1 !)^{\sigma_{1}}(2 !)^{\sigma_{2} \cdots(m !)^{\sigma_{m}}}}$ no of partitions $\eta$ of the set $\{1,2, \cdots, m\}$, contributing same $\partial(\eta)$. Choose such an $\eta$. Then its contribution to (1), that is, $f^{|\eta|}(z) \prod_{B \in \eta} \frac{\partial^{|B|} z}{\prod_{i \in B}^{\partial x_{i}}}$ when evaluated at $x_{1}=x_{2}=\cdots=x_{m}=1$ and remembering that $|\eta|=|\sigma|$, gives

$$
\begin{aligned}
& \frac{n !}{(n-|\sigma|) !}\left(\frac{m q(q+1)^{m}}{2}\right)^{(n-|\sigma|)} \prod_{r=1}^{m} \frac{q^{r \sigma_{r}}(q+1)^{m \sigma_{r}}}{2^{\left(r \sigma_{r}+\sigma_{r}\right)}}\left(\frac{q(3 m+r)+2 r}{3}\right)^{\sigma_{r}} \quad(\text { by }(2),(3)) \\
& =\frac{n !}{(n-|\sigma|) !} \frac{m^{(n-|\sigma|)} q^{(m+n-|\sigma|)}(q+1)^{m n}}{2^{(m+n)}} \prod_{r=1}^{m}\left(\frac{q(3 m+r)+2 r}{r !}\right)^{\sigma_{r}}
\end{aligned}
$$

when $|\sigma|(=|\eta|) \leq n$ and 0 otherwise.

So, finally we have the following

$$
\mathcal{S}=\frac{m ! n !(q+1)^{m n}}{2^{(m+n)}} \sum_{\substack{\sigma \in p(m),|\sigma| \leq m \wedge n}} \frac{m^{(n-|\sigma|)} q^{(m+n-|\sigma|)}}{\sigma_{1} ! \sigma_{2} ! \cdots \sigma_{m} !(n-|\sigma|) ! 3^{|\sigma|}} \prod_{r=1}^{m}\left(\frac{q(3 m+r)+2 r}{r !}\right)^{\sigma_{r}}
$$

This completes the proof of the first part.

Proof of the second part: Let $g(y)=\prod_{i=1}^{m}\left(\sum_{k=0}^{q}\left(x_{i} y\right)\right)$. So

$$
P\left(x_{1}, x_{2}, \ldots, x_{m}, y_{1}, y_{2}, \ldots, y_{n}\right)=\prod_{j=1}^{n} g\left(y_{j}\right) .
$$

Hence

$$
\frac{\partial^{n}}{\partial y_{1} \partial y_{2} \cdots \partial y_{n}} P\left(x_{1}, x_{2}, \ldots, x_{m}, y_{1}, y_{2}, \ldots, y_{n}\right)=\prod_{j=1}^{n} \frac{\partial}{\partial y_{j}} g\left(y_{j}\right)
$$

Again

$$
\frac{g^{\prime}(y)}{g(y)}=\frac{\sum_{k=0}^{q} k x_{i}^{k} y^{k-1}}{\sum_{k=o}^{q} x_{i}^{k} y^{k}} .
$$

Let $X_{i}:=x_{i}+x_{i}^{2}+\cdots+x_{i}^{q}$ and $\bar{X}_{i}:=x_{i}+2 x_{i}^{2}+\cdots+q x_{i}^{q}$. Therefore we have,

$$
g^{\prime}(y)=\left(\prod_{i=1}^{m}\left(1+X_{i}\right)\right)\left(\sum_{i=1}^{m} \frac{\bar{X}_{i}}{1+X_{i}}\right)
$$

when evaluated at $y=1$. Hence, $\frac{\partial^{n}}{\partial y_{1} \partial y_{2} \cdots \partial y_{n}} P\left(x_{1}, x_{2}, \ldots, x_{m}, y_{1}, y_{2}, \ldots, y_{n}\right)$, when evaluated at $y_{1}=y_{2}=\cdots=$ $y_{n}=1$, gives

$$
\left(1+X_{1}\right)^{n}\left(1+X_{2}\right)^{n} \cdots\left(1+X_{m}\right)^{n}\left(\frac{\bar{X}_{1}}{1+X_{1}}+\frac{\bar{X}_{2}}{1+X_{2}}+\cdots+\frac{\bar{X}_{m}}{1+X_{m}}\right)^{n}
$$

Define, $F_{i}:=\left(1+X_{i}\right)^{n} \cdots\left(1+X_{m}\right)^{n}$ and $\mathcal{F}:=\frac{\bar{X}_{1}}{1+X_{1}}+\frac{\bar{X}_{2}}{1+X_{2}}+\cdots+\frac{\overline{X_{m}}}{1+X_{m}}$. Our task is to find $\frac{\partial^{m}}{\partial x_{1} \partial x_{2} \cdots \partial x_{m}} F_{1} \mathcal{F}^{n}$ at $x_{1}=x_{2}=\cdots=x_{m}=1$. Note that,

$$
\frac{\partial}{\partial x_{1}} F_{1} \mathcal{F}^{n}=n\left(1+X_{1}\right)^{n-1} F_{2} \mathcal{F}^{n} \frac{\partial X_{1}}{\partial x_{1}}+n\left(1+X_{1}\right)^{n} F_{2} \mathcal{F}^{n-1} \frac{\partial}{\partial x_{1}}\left(\frac{\bar{X}_{1}}{1+X_{i}}\right) \text {. }
$$

Define $\mathcal{F}_{1}:=\mathcal{F}$ and $\mathcal{F}_{i}:=\mathcal{F}$ evaluated at $x_{1}=x_{2}=\cdots=x_{i-1}=1$ for all $i>1$. Define, $S_{1}:=\left(1+X_{i}\right)^{n-1} \frac{\partial X_{i}}{\partial x_{i}}$ evaluated at $x_{i}=1$ and $S_{2}:=\left(1+X_{i}\right)^{n} \frac{\partial}{\partial x_{i}}\left(\frac{\bar{X}_{i}}{1+X_{i}}\right)$ evaluated at $x_{i}=1$ (It is easy to see that $S_{1}$ and $S_{2}$ are 
well defined, i.e. they do not depend on the choice of $i$ ). In fact, a little computation yields $S_{1}=\frac{q(q+1)^{n}}{2}$ and $S_{2}=\frac{q(q+2)(q+1)^{n}}{12}$.

Now, for any $k \in \mathbb{N}$, we have

$$
\frac{\partial}{\partial x_{i}} F_{i} \mathcal{F}_{i}^{k}=k S_{1} F_{i+1} \mathcal{F}_{i+1}^{k}+S_{2} F_{i+1} k \mathcal{F}_{i+1}^{k-1}
$$

when evaluated at $x_{i}=1$. Suppose, $D$ is an operator, satisfying $D \mathfrak{Q}^{n}=n \mathfrak{Q}^{n-1}$, when $n \geq 0$ and $D \mathfrak{Q}^{n}=0$, when $n<0$, where $\mathfrak{Q}$ is a rational function in $m$ variables. $I$ is defined to be the identity operator on the set of all rational functions in $m$ variables.

So, in the light of (4), the iterated derivative $\frac{\partial^{m}}{\partial x_{1} \partial x_{2} \cdots \partial x_{m}} F_{1} \mathcal{F}^{n}$ at $x_{1}=x_{2}=\cdots=x_{m}=1$, becomes $\left(n S_{1} I+S_{2} D\right)^{m} \mathcal{F}^{n}$ at $x_{1}=x_{2}=\cdots=x_{m}=1$, which is equal to (via a bit computation),

$$
\frac{q^{(m+n)}(q+1)^{m n} m^{n} n^{m}}{2^{(m+n)}} \sum_{i=0}^{m \wedge n} i !\left(\begin{array}{c}
m \\
i
\end{array}\right)\left(\begin{array}{c}
n \\
i
\end{array}\right)\left(\frac{q+2}{3 m n q}\right)^{i} .
$$

\section{Further explore}

The following problems naturally arise from our work.

(1) Can one write down $\xi_{k}$ free formulas for $M(m, n, q, k)$ and $H(n, q, k)$ appeared in Theorem 1.1 and 1.2 respectively? It is worth mentioning that one can actually find $\xi_{k}$ free formulas for these quantities, if $q \equiv 0,1,-1(\bmod k)$ but we have not been able to find a $\xi_{k}$ free formula in general.

(2) We would like to see a combinatorial (or direct algebraic) proof of the identity that occurred in Corollary 1.6. Also, a direct algebraic proof of the identity that occurred in Corollary 2.1 would be of interest.

(3) Also it would be worth exploring whether the techniques of the present paper could be applied to find coefficients or any kind of relations between them for general symmetric polynomials.

\section{Acknowledgements}

The authors would like to thank Prof. Ira Gessel and Prof. R.B. Bapat for their constructive comments and valuable suggestions. The first author would like to thank Prof. Basudeb Datta for his constant support and encouragement. The second would like to thank Dr. Arindam Banerjee and Dr. Kamal Lochan Patra for their constant support and fruitful suggestions towards the overall improvement of this article. The authors would like to thank the anonymous referee for many valuable suggestions which significantly improved the overall exposition of this paper. The first author was supported by NBHM Post Doctoral Fellowship Grant 0204/3/2020/RD-II/2470. The second author was supported by the Council of Scientific and Industrial Research grant no. 09/1248(0004)/2019-EMR-I, Ministry of Human Resource Development, Government of India.

\section{References}

[1] M. Abramson and W. O. J. Moser, Arrays with fixed row and column sums, Discrete Math. 6 (1973), 1-14.

[2] H. Anand, V. C. Dumir, and H. Gupta, A combinatorial distribution problem, Duke Math. J. 33 (1966), 757-769.

[3] L. Carlitz, Enumeration of symmetric arrays, Duke Math. J. 33 (1966), 771-782.

[4] L. Carlitz, Enumeration of symmetric arrays. II, Duke Math. J. 38 (1971), 717-731.

[5] I. M. Gessel, Symmetric functions and P-recursiveness, J. Combin. Theory Ser. A 53:2 (1990), 257-285.

[6] I. P. Goulden and D. M. Jackson, Labelled graphs with small vertex degrees and P-recursiveness, SIAM J. Algebraic Discrete Methods 7:1 (1986), 60-66.

[7] H. Gupta, Enumeration of symmetric matrices, Duke Math. J. 35 (1968), 653-659.

[8] M. Hardy,Combinatorics of partial derivatives, Electron. J. Combin. 13:1 (2006), \#R1. 